\section{Laquihuanaco-Coarita GM ${ }^{1}$ Rodríguez-Cárdenas YA ${ }^{2}$ Ruíz-Mora $\mathrm{GA}^{3}$}

Maestría en Radiología Bucal y Maxilofacial, Escuela de Odontología, Universidad Científica del Sur-UCSUR, Lima, Perú.

Profesor Internacional de la Maestría en Radiología Bucal y Maxilofacial, Escuela de Odontología, Universidad Científica del Sur. 3. Maestría en Radiología Bucal y Maxilofacial, Escuela de Odontología, Universidad Científica del Sur-UCSUR, Lima, Perú. Profesor Asociado a la División de Ortodoncia, Facultad de Odontología, Universidad Nacional y Universidad El Bosque Bogotá, Colombia.

\section{ARTÍCULO ORJGINAL}

\title{
HALLAZGO RADIOLÓGICO INCIDENTAL: CÓNDILO BÍFIDO, CALCIFICACIÓN DE LIGAMENTO ESTILOHIOIDEO Y TORUS MANDIBULAR. REPORTE DE CASO
}

\section{RESUMEN}

El presente reporte de caso describe un hallazgo radiológico incidental simultáneo de cóndilo bífido, calcificación de ligamento estilohioideo y torus mandibular. Un estudio radiológico de rutina para fijación de implantes óseointegrados en una mujer de 56 años en el Centro de Diagnóstico por Imágenes de Lima-Perú, con imágenes panorámica y tomografía computarizada de haz cónico (TCHC), permitió encontrar incidentalmente alteraciones morfológicas unilaterales del cóndilo mandibular y apófisis estiloides derechos, además de un torus mandibular lingual bilateral.

PALABRAS CLAVE: Hallazgos incidentales, cóndilo mandibular, variación anatómica, TCHC

INCIDENTAL RADIOLOGICAL FINDING: BIFID CONDYLE, CALCIFICATION OF THE STYLOHYOID LIGAMENT AND MANDIBULAR TORUS. A CASE REPORT

\section{ABSTRACT}

This case report describes a simultaneous incidental radiological finding of bifid condyle, calcification of the stylohyoid ligament and mandibular torus. A routinary radiological study for fixation of osseointegrated implants in a woman of 56 years old at the Centro de Diagnóstico por Imágenes in Lima-Perú, with panoramic imaging and cone beam computed tomography (CBCT), allowed to find incidentally unilateral morphological changes of the right mandibular condyle and right styloid process as well as a bilateral lingual mandibular torus. seconds.

KEY WORDS: Incidental findings, mandibular condyle, anatomical variation, CBCT 


\section{INTRODUCCIÓN}

Los hallazgos incidentales son aquellos detectados por azar, son un evento raro pero significativo. Es importante para el clínico buscar otras anormalidades de las que esperan encontrar y estar preparados para interpretar y comunicar estos hallazgos.

El cóndilo bífido es una anomalía rara, observada frecuentemente como hallazgo radiográfico, caracterizada por la división de la cabeza condilar. La primera vez que se describió esta alteración fue en el American Journal of Physical Anthropology en 1941 por Hrdlicka. ${ }^{1}$ Su prevalencia varía entre $0.0018 \%$ a $0.3 \% .^{2,3}$

No parece existir una predilección especial de aparición en una raza o sexo y en la mayoría de los casos no hay clínica de disfunción de la articulación temporomandibular. Los casos suelen ser unilaterales ${ }^{4-7}$

Las dos superficies articulares del cóndilo bífido pueden ser divididas por un surco orientado mediolateralmente o anteroposteriormente. La división del cóndilo puede variar desde un surco superficial hasta la formación de dos cabezas de cóndilo distintas. ${ }^{3,4,6}$

Su etiología y patogenia se desconocen. Se cree que es una alteración del desarrollo debido a un traumatismo o a desórdenes endocrinos, farmacológicos o nutricionales. ${ }^{8}$ Tampoco se conoce la relevancia clínica que puede suponer presentar esta alteración, puesto que hay casos en los que sí se aprecian cambios artríticos en la articulación. ${ }^{8}$

La mineralización patológica de los tejidos blandos puede aparecer en una amplia variedad de trastornos y procesos degenerativos relacionados. ${ }^{9-11}$

Eagle $^{12}$ señaló que la longitud normal de la apófisis estiloides es de $25 \mathrm{~mm}$. El alargamiento de la apófisis estiloides es común y casi siempre resulta de la osificación de los ligamentos estilohioideo y/o estilomandibular. La mineralización o calcificación de la cadena estilohioidea es relativamente común. ${ }^{10-14}$
La elongación y calcificación del complejo de la cadena estilohioidea se observan fácilmente en las radiografías panorámicas. La literatura reporta una prevalencia entre el $4 \%$ y $28 \%$ de la población. ${ }^{11-14}$

Las exostosis también conocida como hiperostosis, son protuberancias óseas. Son más comunes en mujeres, su incidencia varía desde 0.5 a $85 \%$ y es encontrada en habitantes de ciudades del norte del Perú. ${ }^{9}, 10,15,16$

\section{REPORTE DE CASO}

Se presenta una paciente mujer de 56 años de edad, que acudió al Centro de Diagnóstico por Imágenes Lima-Perú, para realizarse estudios radiográficos previos a su tratamiento odontológico (fijación de implantes oseointegrados).

En la radiografía panorámica (Figura 1) se observó la alteración de forma en el cóndilo derecho y calcificación del proceso estilohioideo también del lado derecho. Todos fueron hallazgos radiográficos incidentales. La paciente no reportó sintomatología ni antecedentes de traumatismo previo. Del mismo modo, al examen físico no fueron observadas limitaciones de apertura bucal o la presencia de sonidos articulares. Se realizó una adquisición con tomógrafo Picasso Master $3 \mathrm{D}$, Vatech Hwaseong, Corea del Sur, FOV $20 \mathrm{~cm}$ $\mathrm{x} 19 \mathrm{~cm}$, de 18 segundos, $8 \mathrm{~mA}$ y $90 \mathrm{kV}$, solicitado por un odontólogo para fijación de implantes oseointegrados. Los cortes axial (Figura 2), coronal (Figura 3), sagital (Figura 4) y reconstrucción volumétrica VR (Figura 5) muestran en detalle las variantes anatómicas compatibles con cóndilo bífido y calcificación del ligamento estilohioideo.

En la vista axial se evidenció también la presencia de torus lingual bilateral. (Figura 6)En vista de que la paciente no presentaba signos/síntomas, ningún tratamiento fue instaurado.

\section{DISCUSIÓN}

La literatura sugiere varios orígenes para el desarrollo del cóndilo bífido, sin embargo no hay acuerdo entre los estudios acerca del factor causante principal..$^{3-8,10,17} \mathrm{Se}$ cree que el cóndilo bífido 


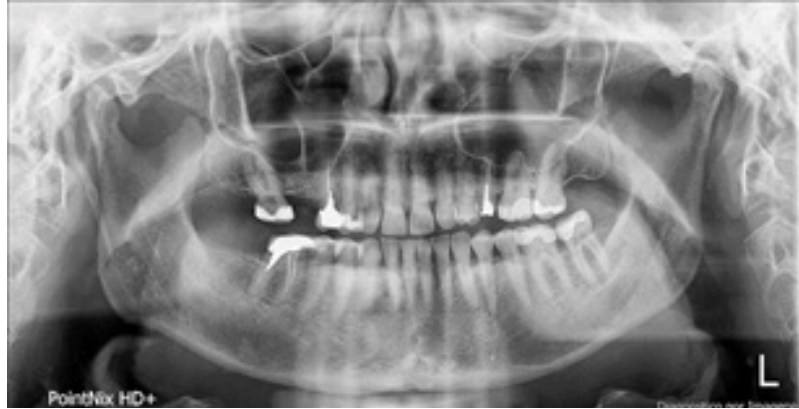

Figura 1. Imagen panorámica digital que muestra alteración morfológica aparente de la cabeza condilar derecha y calcificación del ligamento estilohiodeo derecho.

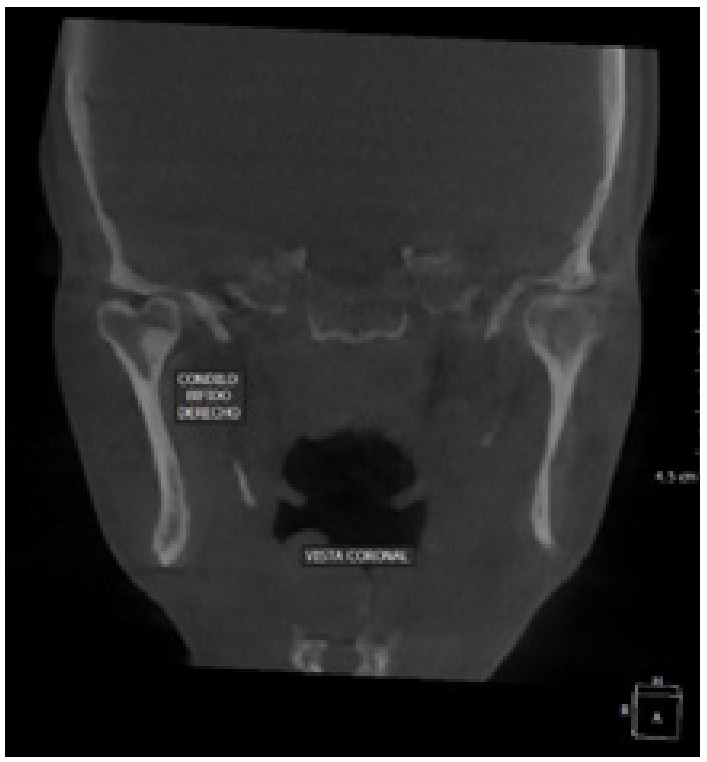

Figura 2. Corte axial donde se observa la imagen de cóndilo bifido derecho.

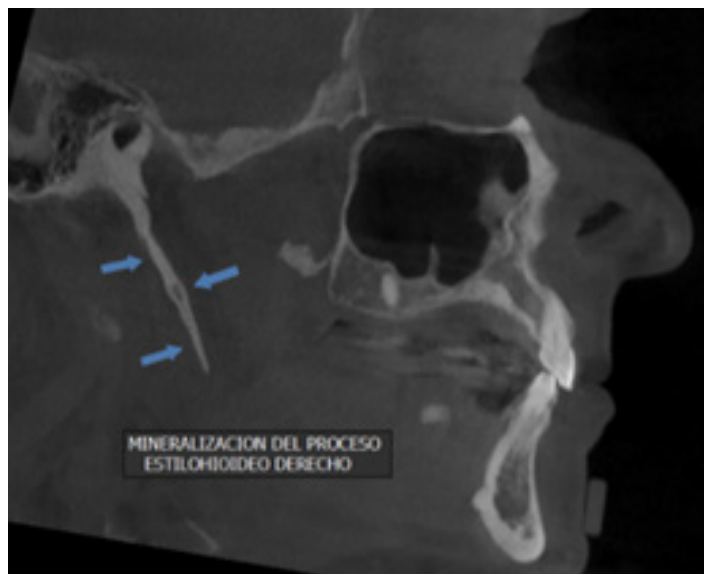

Figura 3. Corte coronal que muestra la imagen de cóndilo bifido derecho(flecha).

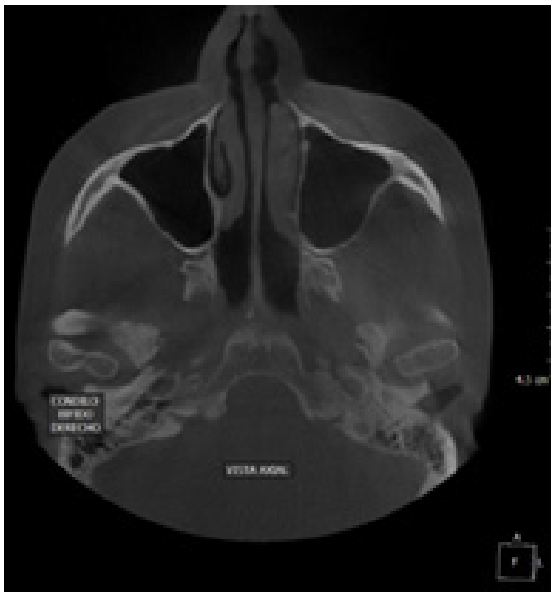

Figura 4. Corte sagital que evidencia la calcificación del ligamento estilohioideo derecho.

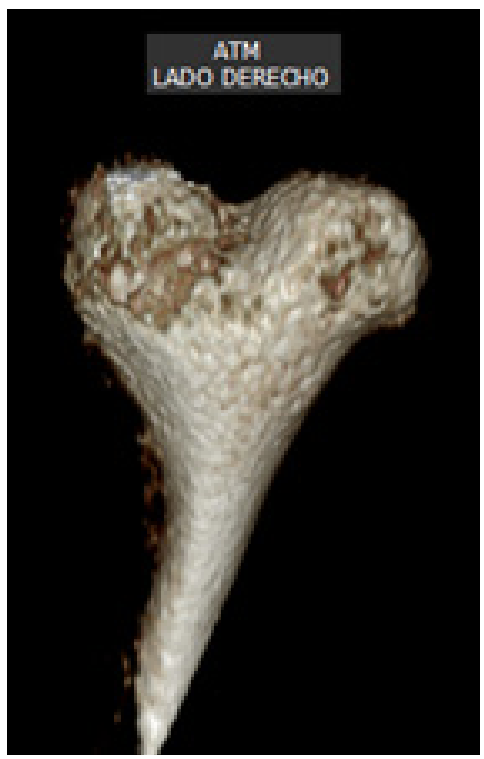

Figura 5. Reconstrucción volumétrica del cóndilo derecho que muestra los rasgos típicos del cóndilo bífido.

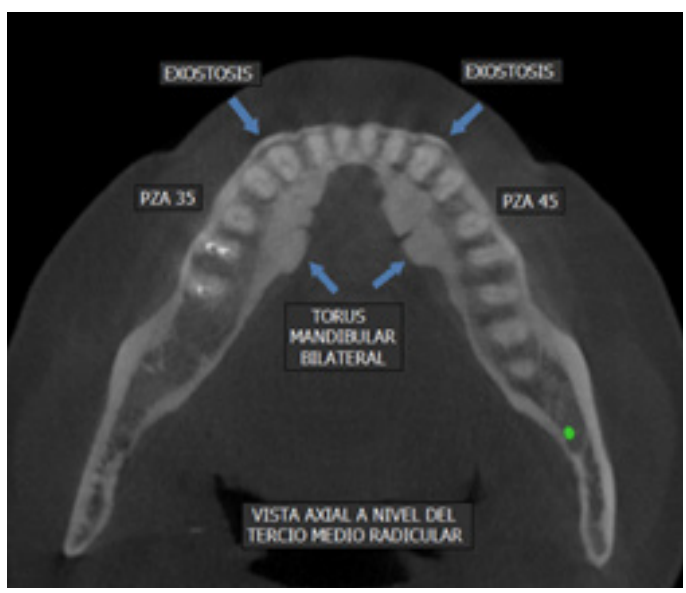

Figura 6. Corte axial donde se observa imagen hiperdensa bilateral de bordes definidos compatible con torus mandibular bilateral. 
es una anomalía del desarrollo. Ha sido clasificada como de origen genético primario. Sin embargo, diferentes cuadros clínicos describen causas secundarias adicionales, como el traumatismo perinatal, la fractura condilar o la condilectomía quirúrgica. En otros casos no hay historia de traumatismos o síntomas asociados aunque la anquilosis puede estar relacionada. . $^{3-10}$

Antoniades et $a l^{8}$ sugirieron que el desarrollo del cóndilo mandibular bífido es causado debido a una capacidad de remodelación ósea insuficiente. Quayle y Adams ${ }^{18}$, indicaron que los desórdenes endocrinos, deficiencia nutricional, infección, trauma, irradiación y factores genéticos pueden ser posibles factores causales. ${ }^{6}$ Thomason y Yusul $^{19}$ relataron dos casos de fractura condilar de origen traumático, por accidente de bicicleta, que acarreó en la formación de un cóndilo bífido.

En el presente caso, la paciente no relató ningún antecedente de traumatismos ni presentó ningún tipo de sintomatología, sin embargo no se puede asegurar el origen del cóndilo bífido.

La epidemiología de esta alteración es desconocida puesto que en la mayoría de los casos es asintomática y es diagnosticada en radiografías panorámicas de rutina. Sin embargo, Menezes et $a l^{2}$ examinó 50800 radiografías panorámicas de la población brasilera, observando 9 casos $(0.0018 \%), 7$ unilaterales y 2 bilaterales, considerándose una baja prevalencia de cóndilo bífido.

No hay predilección por algún sexo, raza o grupo etáreo. ${ }^{5,6}$ Parecen ser más comunes en el lado izquierdo en casos unilaterales en una proporción de 2:1. ${ }^{7}$ En el caso reportado, el hallazgo radiográfico evidenció cóndilo bífido de lado derecho.

La morfología puede variar de un surco superficial a la presencia de dos cabezas condilares distintas. Se ha postulado que la división anteroposterior ocurre generalmente en pacientes con antecedentes de trauma en la infancia mientras que el sentido medio-lateral se desarrolla debido a la permanencia de un tabique o septo fibroso en el cartílago condilar. ${ }^{3,4,6}$ En el presente caso en la tomografía computarizada de haz cónico se evidenció una división anteroposterior de la cabeza condilar, sin embargo, la paciente no reportó historia de trauma en la infancia, corroborando así la postulación de Cowan y Fergusson ${ }^{20}$, quienes indicaron que el factor causante no tiene influencia en el sentido del cóndilo bífido.

Cuando la sintomatología está presente, los más comunes son sonidos articulares, raramente dolor articular y anquilosis. Generalmente no hay sintomatología presente, como en el caso reportado, por lo que el diagnóstico de un cóndilo bífido frecuentemente se apoya más en una evidencia radiográfica que en la clínica. ${ }^{4-8,21}$

En el diagnóstico de esta variante anatómica, la radiografía panorámica suele ser la primera evidencia, no obstante la tomografía computarizada de haz cónico es más definitoria y la herramienta de elección, ya que puede aportar mayor información de la morfología condilar y ayuda al profesional a hacer el diagnóstico diferencial frente a otras patologías de la ATM como tumores y quistes primarios óseos, lesiones metastásicas, lesiones óseas degenerativas y lesiones metabólicas que también pueden alterar la morfología condilar. ${ }^{3-6,21}$

Tradicionalmente se observaron los cóndilos bífido mediante exámenes de radiología convencional; durante los últimos años, gracias al advenimiento de la tomografía de haz cónico, ha sido posible la evaluación más detallada de la morfología condilar sin la superposición de otras estructuras anatómicas. ${ }^{6,21}$

El tratamiento apropiado para los casos de cóndilo bífido depende exclusivamente de los síntomas presentados por el paciente. ${ }^{5,8}$ Para los casos sintomáticos a veces requieren de cirugía; suelen ser casos secundarios a traumatismos con anquilosis condilar aunque la gran mayoría son asintomáticos o presentan grados leves de disfunción de ATM que responden a un tratamiento conservador de antinflamatorios, relajantes musculares, dieta blanda y férula de descarga..$^{3-6,8,10,11,21}$ En el presente caso, debido a la ausencia de sintomatología en la paciente, no se instauró ningún tipo de tratamiento. 
La calcificación del proceso estilohioideo como hallazgo radiográfico ha sido descrito por varios autores como un acontecimiento que clínicamente puede ser asintomático o manifestarse con diferentes tipos de signos y síntomas que incluso pueden llevar a diferentes síndromes. ${ }^{11,13}$ Cuando los síntomas están presentes, la afección se conoce como Síndrome de Eagle, Síndrome estilohioideo y/o neuralgia de la apófisis estiloides. ${ }^{10-14}$

En el presente caso, se observa imagen radiopaca proyectada desde borde inferior del conducto auditivo externo a tercio medio externo de rama mandibular derecha de bordes continuos y definidos, imagen compatible con calcificación parcial no segmentada del ligamento estilohioideo, sin presentar ningún tipo de sintomatología, por lo que es considerado como el segundo hallazgo radiográfico.

El torus mandibular es una exostosis conocida también como hiperostosis, se presenta como una protuberancia benigna que se levanta sobre la lámina central. Su etiología no es clara, probablemente puede ser causada por la acción conjunta de factores genéticos, medio ambientales y la hiperfunción masticatoria. La mayoría de los torus son encontrados entre los 11 a 30 años y son raros antes de los 10 años. Menos del 3\% ocurre en niños. Los torus pueden ser prominentes o multilobulados pero mayormente son únicos. No requieren tratamiento, a menos que sean grandes $\mathrm{o}$ alteren la función. ${ }^{10,15,16}$

En el caso reportado, se observó tanto en la radiografía panorámica como en los cortes tomográficos, la presencia de torus mandibular bilateral, asintomático, considerándose el tercer hallazgo radiográfico. Hotta et $a l^{22}$ reportaron un caso de síndrome de Eagle y torus mandibular en una mujer de 62 años, concluyendo que ambas alteraciones sumadas a una actividad parafuncional tienen una alta prevalencia en pacientes con disfunción de la articulación temporomandibular y podrían ser útiles como un indicador de aumento de riesgo de los trastornos temporomandibulares. ${ }^{22}$

La tomografía computarizada de haz cónico es una técnica radiográfica tridimensional que es comúnmente utilizada en el diagnóstico y el plan de tratamiento en diversos campos de la odontología. Los hallazgos incidentales son frecuentemente reportados en la literatura. Rheem et $a l^{23}$ reportaron de un total de $147 \mathrm{TCHC}$, una tasa global de hallazgos incidentales de 40,1\%. El rango de edad de los pacientes fue de 8 a 80 años. La mayor tasa de hallazgos incidentales se encontraba en la región sinusal (51.7\%), seguido por la región dentoalveolar $(34.01 \%)$, región de ATM (26,53\%), región ósea (15,64\%), calcificación de ligamentos, glándula pineal y arteria carótida (12,92\%), anomalías dentales (10,88\%), región nasal $(8.84 \%)$ y región de las vías aéreas $(5.44 \%) .^{23}$

\section{CONCLUSIÓN}

1. Los hallazgos radiográficos en esta paciente fueron asintomáticos incluyendo el cóndilo bífido, la calcificación del ligamento estilohioideo y el torus mandibular bilateral.

2. Los hallazgos fueron incidentales durante exámenes panorámicos de rutina, sin embargo, las imágenes tomográficas son determinantes en el diagnóstico final y en caso de ser solicitadas deben ser evaluadas de forma multiplanar y con una revisión cuidadosa.

\section{REFERENCIAS BIBLIOGRÁFICAS}

1. Hrdlicka A. Lower jaw: Double condyles. Am J Phys Anthropol. 1941;28:75-89.

2. Menezes A, De Moraes F, De VasconcelosFilho J, Kurita L, De Almeida S, HaiterNeto F. The prevalence of bifid mandibular condyle detected in a Brazilian population. Dentomaxillofac Radiol.2008; 37(4):220-3.

3. Neves F, Ramirez L, Freitas D, Haiter-Neto F, Bóscolo F. Cóndilo Mandibular Bífido. Un desafío diagnóstico. Int J Odontostomat. 2012;6(3): 327-30.

4. Faisal M, Ali I, Pal U, Bannerjee. Bifid mandibular condyle: Report of Two cases of varied etiology. Natl J Maxillofac Surg. 2010; 1(1):78-80. 
5. Corchero G, Gonzáles T, García M, Sánchez S, Saiz R. Bifid Condyle: Case Report. Med Oral Patol Oral Cir Bucal. 2005;10:277-9.

6. Melo S, Barbosa J, Peixoto A, Santos T, Gerbi M. Bilateral Bifid Mandibular Condyle: A Case Report. Int JMorphol.2011;29(3):922-6.

7. Loh F, Yeo J. Bifid mandibular condyle. Oral Surg Oral Med Oral Pathol. 1990; 69:24-7.

8. Antoniades K, Hadjipetrou L, Antoniades V, Paraskevopoulos K. Bilateral bifid mandibular condyle. Oral Surg Oral Med Oral Pathol Oral Radiol Endod. 2004; 97:535-8.

9. Langlais R. Diagnostic Imaging of the jaws. Malvern. Editorial Williams and Wilkins. 1995.

10. Goaz P, White S. Radiologia Oral. 3ra ed. Madrid. Mosby.1995.

11. Gulhal Bozkir M, Boga H, Dere F. The Evaluation of Elongated Styloid Process in Panoramic Radiographs in edentulous Patients. Journal of medical Science. 1999;29:481-5.

12. Eagle WW. Elongated styloid process: report of two cases. Arch Otolaryngol. 1937; 25:584-7.

13. Rubio C, Ganga H, Guzmán C. Análisis de la Longitud del Ligamento Estilohiodeo Mediante Sistema Cone Beam. Revista CES Odontología. 2010;23(1):23-7.

14. Monsour P, Young W. Variability of the styloid process and stylohyoid ligament in panoramic radiographs. Oral Surg Oral Med Oral Pathol. 1986;61(5):522-6.

15. Sangwan A, Sharma K. Mandibular tori. A case report and review. Int Journal of Contemporary Dentistry. 2011;2(5):125-7.
16. Meza J. Cavidad oral: Torus palatinus y torus mandibulares. Rev Gastroenterol Perú. 2004; 24:343-8.

17. Stefanou E, Fanourakis I, Vlastos K, Katerelou J. Bilateral bifid mandibular condyles. Report of four cases. Dentomaxillofac Radiol. 1998; 27:186-8.

18. Quayle A, Adams J. Supplemental mandibular condyle. Br J Oral Maxillofac Surg. 1986;24(5):349-56.

19. Thomason J, Yusul H. Traumatically induced bifid mandibular condyle: a report of two cases. Br Dent J.1986;161(8):291-3.

20. Cowan D, Ferguson M. Bifid mandibular condyle. Dentomaxillofac. Radiol.1997;26(1):70-3.

21. Millas R, Cajas J, Causa M, Melo I, Casals M, Brunetto L y col. Cóndilos bífido y trífido en disfunción de la articulación temporomandibular: Reporte de dos casos clínicos. Revista Chilena de Radiología. 2010;16(4):169-74.

22. Hotta T, Sverzut C, Palinkas M, Bataglion C, Melchior M, Hotta P et al. Case report involving temporomandibular dysfunction, eagle's síndrome and torus mandibularis - Multidisciplinary approaches. Open J Stomatology. 2013;3:338-43.

23. Rheem S, Nielsen IL, Oberoi S. Incidental findings in the maxillofacial region identified on cone-beam computed tomography scans. J Orthod Res. 2013;1:33-9. 